\title{
Influence of Gamma-Hydroxybutyric Acid-Use and Gamma-Hydroxybutyric Acid-Induced Coma on Affect and the Affective Network
}

\author{
Filipa Raposo Pereira ${ }^{a}$ b Minni T.B. McMaster ${ }^{a}$ b Yvon D.A.T. de Vries $^{a}$ \\ Nikki Polderman ${ }^{a}$ Wim van den Brink ${ }^{a, b}$ Guido A. van Wingen ${ }^{a, b}$ \\ a Department of Psychiatry, Amsterdam Neuroscience, Amsterdam UMC, University of Amsterdam, Amsterdam, \\ The Netherlands; ${ }^{\mathrm{b}}$ Amsterdam Brain and Cognition, University of Amsterdam, Amsterdam, The Netherlands
}

\section{Keywords}

Anxiety · Emotion identification · Gamma-hydroxybutyric acid - Gamma-hydroxybutyric acid-induced coma, neuroimaging $\cdot$ Substance of addiction

\section{Abstract}

Background: Gamma-hydroxybutyric acid (GHB) is a drug of abuse associated with increased emergency room attendances, due to GHB-induced comas. Withdrawal from GHB often increases social anxiety and is linked to alterations in emotion processing. However, little is known about the effects of GHB-use and GHB-induced comas on affect regulation in humans. Objectives: We aimed to assess the effect of GHB-use and GHB-induced comas on the affective network. Method: We recruited $27 \mathrm{GHB}$ users with $\geq 4 \mathrm{GHB}$-induced comas (GHB-Coma), 27 GHB users without a GHB-induced coma (GHB-NoComa), and 27 polydrug users who never used GHB (No-GHB). Participants completed self-report questionnaires assessing negative affect (depression, anxiety and stress) and performed an emotional face matching task during functional magnetic resonance imaging to probe activity of the amygdala and the hippocampus. Results: The GHB-Coma group reported higher levels of depression, anx-

\section{KARGER}

E-Mail karger@karger.com www.karger.com/ear

\section{Karger \\ Published by S. Karger AG, Basel Open access}

This article is licensed under the Creative Commons AttributionNonCommercial-NoDerivatives 4.0 International License (CC BY NC-ND) (http://www.karger.com/Services/OpenAccessLicense). Usage and distribution for commercial purposes as well as any distribution of modified material requires written permission. iety, and stress; showed decreased activity of the hippocampus; and increased functional connectivity of the left hippocampus with the left fusiform gyrus and a cluster on the left temporal-parietal-occipital junction, when compared with the 2 other groups. The GHB-NoComa group showed decreased functional connectivity of the left hippocampus with the amygdala in comparison with the No-GHB group. Conclusions: GHB-use but in particular GHB-induced comas, are associated with altered emotion identification and hippocampal functioning. Awareness campaigns are required to raise consciousness about the adverse effects of GHB-induced comas on affect regulation, despite the absence of subjective side effects.

(c) 2019 The Author(s)

Published by S. Karger AG, Basel

\section{Introduction}

Regardless of the relative low prevalence of use, intoxication with Gamma-hydroxybutyric acid (GHB) is currently the fourth most common recreational drug-related intoxication presented at emergency rooms in Europe [14]. These emergency attendances predominantly resulting from $\mathrm{GHB}$-induced comas, are paralleled by growing 
numbers of users seeking treatment for GHB dependence [3-6]. Recreational GHB-use is known to elicit sociability and emotional awareness, and acute GHB exposure has been linked to altered brain activity of regions related to emotion processing $[1,7,8]$. However, the long-term effects of regular GHB-use and GHB-induced comas on the affective network have not yet been assessed.

Withdrawal from recreational or medical use of GHB is often associated with anxiety, stress, and sporadically depression [6, 9-13]. Moreover, regular use of GHB has been associated with persistent alterations in emotion identification, decreased social interaction, and increased social anxiety $[10-12,14,15]$. These are negative affects regulated by a major affective network, where the amygdala and the hippocampus are central processing hubs $[16,17]$. Interestingly, animal studies show that these are regions particularly sensitive to neurotoxic effects induced by GHB, and more, we found that regular use of GHB is also associated with differences in hippocampus processing in humans $[10,15,18-20]$.

GHB has a narrow dose margin between the desired effects and overdose $[2,21]$. As a result, many regular users of GHB experience multiple GHB-induced comas lasting between 1 and $4 \mathrm{~h}$ and often scoring the most critical classification on the Glasgow coma scale $[1,2,9,21]$. Even if transient, these comas have been associated with hypoxia (oxygen deprivation) and may lead to oxidative stress in the amygdala and the hippocampus [22-24]. However, since no negative outcomes are experienced immediately after returning to consciousness from $\mathrm{GHB}$ induced comas, most users still believe that GHB use is safe and these comas are harmless $[1,2,25]$.

Despite the potential negative effects of regular $\mathrm{GHB}$ use, studies assessing the influence of GHB-use and multiple GHB-induced comas on the affective network are lacking. We used self-report questionnaires to assess negative affect and an emotional face matching task during functional magnetic resonance imaging (fMRI) to probe amygdala and hippocampus activity. Three groups were recruited to distinguish the effects of GHB use alone and GHB-induced comas: (1) GHB users with $\geq 4$ GHB-induced comas, (2) GHB users without GHB-induced comas, and (3) polydrug users who never used GHB. We aimed to test the following hypotheses: (a) GHB users who had multiple GHB-induced comas report more negative affect and show altered amygdala and hippocampus activity and functional connectivity when compared with GHB users without GHB-induced comas and polydrug users who never used GHB; (b) GHB users without GHBinduced comas report more negative affect and show al- tered amygdala and hippocampus activity and functional connectivity when compared with the polydrug users who never used GHB.

\section{Methods}

\section{Participants}

In this cross-sectional neuroimaging study, 81 male participants were recruited via addiction centers in the Netherlands, flyers, Internet advertisements, and snowball sampling. We engaged 3 different groups of participants matched for age and education level: 27 GHB users with $\geq 4$ GHB-induced comas (GHB-Coma); 27 GHB users without GHB-induced comas (GHB-NoComa); and 27 polydrug users who never used GHB (No-GHB). All groups were polydrug users, since recreational GHB is predominantly used in a polydrug context. Inclusion criteria for all groups were age between 18 and 40 years, native Dutch speakers and since the majority of GHB-users are males, we only included men [9]. Inclusion for GHB users also consisted of the use of GHB $\geq 25$ times in the last 2 years preceding this assessment. Inclusion for the GHBComa group also consisted of the arbitrary minimum of 4 GHBinduced comas in order to increase the contrast with the GHBNoComa group. We defined polydrug use as the use of alcohol, tobacco, cannabis, cocaine, any other stimulants (amphetamines, khat, methylphenidate), ecstasy, ketamine, and sedatives (benzodiazepines). Exclusion criteria for all participants were a history of epilepsy, general anesthesia in the 2 years preceding the study; a contraindication for fMRI scanning (e.g., metal objects in the body or head injury); any coma not related to GHB use; and currently under treatment for narcolepsy with cataplexy (since treatment may involve the use of medical GHB). Participants were required to be abstinent from recreational drugs for at least $24 \mathrm{~h}$ preceding the beginning of this study. MRI data of 3 GHB-Coma participants, 3 GHB-NoComa participants, and 1 No-GHB participant were discarded due to excessive head movement inside the scanner and/or insufficient brain coverage.

After explaining the study procedure, written informed consent was obtained from all participants before study initiation. The study was in accordance with the Helsinki Declaration principles (7th revision, 2013), the Medical Research Involving Human Subjects (WMO, 1998), and approved by the Medical Ethics Review Committee of the Academic Medical Centre [26, 27].

\section{Procedure}

The study consisted of an initial urine test, followed by completing questionnaires related to GHB and other drug use, depression, anxiety, stress, and impulsivity levels. During the subsequent neuroimaging session, structural and functional scans were made in the following order: structural MRI; resting-state fMRI; longterm memory (paired association task) fMRI; diffusion weighted imaging, working memory (n-back task) fMRI; emotion processing (face-emotion matching task) fMRI. Finally outside the scanner, participants performed digitized neuropsychological testing including verbal memory, spatial memory, intra-extra dimensional set shifting, and probabilistic reversal learning. In this report, we present the data related to emotion processing; results from other experiments will be presented elsewhere [19]. 
Table 1. Demographic and clinical data

\begin{tabular}{|c|c|c|c|c|}
\hline & $\begin{array}{l}\text { GHB-coma }(n=24), \\
\text { mean } \pm \mathrm{SD}\end{array}$ & $\begin{array}{l}\text { GHB-NoComa }(n=24) \\
\text { mean } \pm \text { SD }\end{array}$ & $\begin{array}{l}\text { No-GHB }(n=26) \\
\text { mean } \pm \text { SD }\end{array}$ & $\begin{array}{l}\text { Difference, } \\
p \text { value }\end{array}$ \\
\hline Age, years & $25.67 \pm 5.54$ & $26.33 \pm 4.72$ & $27.38 \pm 9.32$ & $0.676^{\mathrm{a}}$ \\
\hline Educational level & $4.88 \pm 1.62$ & $5.25 \pm 1.51$ & $5.27 \pm 1.54$ & $0.611^{\mathrm{a}}$ \\
\hline Premorbid IQ & $89.63 \pm 10.59$ & $98.08 \pm 7.67$ & 93.73 & $0.006^{\mathrm{a}, 1}$ \\
\hline Daily dose, $\mathrm{mL} /$ day & $46.63 \pm 37.48$ & $18.68 \pm 11.01$ & - & $<0.001^{\mathrm{b}}$ \\
\hline Days of GHB use in preceding 30 days & $12.00 \pm 12.81$ & $2.85 \pm 2.20$ & - & $0.044^{\mathrm{b}}$ \\
\hline Months of daily use & $24.96 \pm 42.15$ & $2.85 \pm 2.20$ & - & $0.013^{\mathrm{b}}$ \\
\hline Depression & $1.17 \pm 1.17$ & $0.46 \pm 0.78$ & $0.42 \pm 1.10$ & $0.014^{\mathrm{c}, 1,2}$ \\
\hline Anxiety & $1.21 \pm 1.25$ & $0.29 \pm 0.86$ & $0.19 \pm 0.80$ & $<0.001^{\mathrm{c}, 1,2}$ \\
\hline Stress & $1.00 \pm 1.41$ & $0.04 \pm 0.20$ & $0.15 \pm 0.46$ & $0.001^{\mathrm{c}, 1,2}$ \\
\hline $\begin{array}{l}{ }^{*} p<0.05 . \\
\text { a Analysis of variance. } \\
{ }^{b} \text { Mann-Whitney U. } \\
{ }^{\mathrm{c}} \text { Kruskal-Wallis. } \\
{ }^{1} \text { Post-hoc analysis Mann-Whitney } \\
{ }^{2} \text { Post-hoc analysis Mann-Whitney } \\
\text { GHB, gamma-hydroxybutyric acid. }\end{array}$ & $\begin{array}{l}: \text { GHB-Coma }>\text { GHB-I } \\
\text { : GHB-Coma > No-GH }\end{array}$ & $\begin{array}{l}\text { Coma; depression, } p=0.0 \\
\text {; depression, } p=0.010 \text {; an }\end{array}$ & $\begin{array}{l}\text { anxiety, } p=0.002 ; \text { st } \\
y, p=0.010 ; \text { stress, } p\end{array}$ & $\begin{array}{l}\mathrm{s}, p=0.002 \\
0.001\end{array}$ \\
\hline
\end{tabular}

\section{Clinical Assessment}

As a proxy for IQ, premorbid intellectual functioning was assessed with the Dutch version of the National Adult Reading test [28]. Co-use of other recreational drugs was assessed with the substance use section of the MATE 2:1 questionnaire [29]. Negative affect was assessed using the self-report depression, anxiety, and stress scale-21 [30]. The depression, anxiety, and stress scale consists of 3 clusters of 7 statements each. The statements are rated from 0 to 3 , from not applying to accurately applying to the individual, respectively. The sum of the final score of each affect (depression, anxiety or stress) is then evaluated according to their severity on a scale of 5 levels, normal, mild, moderate, severe, or extremely severe.

\section{Statistical Analysis}

SPSS24 (IBM Software Analytics, New York, NY, USA) was used to assess the demographic and clinical data. Analysis of variance was used for the analysis of normally distributed data. Not normally distributed data were transformed or analyzed with nonparametric tests (Tables 1,2). Co-use of alcohol, tobacco, cannabis, cocaine, other stimulants, ecstasy, ketamine, and sedatives (MATE: 2.1) was assessed by self-reported measures considering the number of days of use in the preceding 30 days, typical daily dose, and the number of years of weekly use of each drug. From these measures, a total exposure variable was defined as years of weekly use $\times$ daily dose for each drug and presented in Table. 2. Daily GHB dose (mL/day), months of daily GHB use, and prevalence of days using GHB in the previous month were analyzed for differences between the GHB groups. Differences between groups were further assessed for depression, anxiety, or stress levels.

\section{fMRI Assessment: Emotional Face Matching Task}

Participants performed an emotional face matching task, known to probe amygdala and hippocampus activity [31, 32]. The block design consisted of the alternated presentation of 2 emo- tional condition blocks (fearful and angry face expressions) with 3 control condition blocks (blurred ellipses). Each block consisted of 6 trials; each trial was presented for 5 seconds with an overall task duration of $2.5 \mathrm{~min}$. Each trial consisted of 3 stimuli presented in triangular positioning, 1 on top, and 2 at the bottom. Only one of these 2 stimuli was similar to the one on top. In the emotional condition, 2 angry faces and a fearful 1 or vice-versa were presented, and participants were requested to identify which of the emotional face expressions on the bottom row matched the one on top using a button press. The stimuli consisted of intermixed male and female faces. In the control condition, 2 blurred horizontal ellipses and a vertical one were presented or vice-versa, and participants were requested to identify which of the bottom ellipses were oriented in the same direction as the one on top.

\section{fMRI Data Acquisition}

MRI data were collected with a 3.0T Ingenia scanner (Phillips Medical System, Best, The Netherlands), with a 32 channel head coil. T1-weighted structural images $\left(1 \times 1 \times 1 \mathrm{~mm}^{3}\right.$ voxels $)$ were acquired for spatial normalization purposes. T2* blood oxygenated level dependent contrast images were collected using an echoplanar sequence, with each volume comprising 37 ascending slices $(\mathrm{TR} / \mathrm{TE}=2,000 / 27 \mathrm{~ms} ; 3 \times 3.08 \times 3 \mathrm{~mm})$.

\section{fMRI Data Analysis}

Neuroimaging data were analyzed with statistical parametric mapping 12 (SPM12; Wellcome Trust Centre for Neuroimaging, London, UK). Functional images were realigned to the mean image, corrected for slice time acquisition, coregistered to the structural scan, spatially normalized into Montreal Neurologic Institute space using the default segmentation procedure implemented in SPM12, resampled into $2 \times 2 \times 2 \mathrm{~mm}^{3}$ voxels, and spatially smoothed with a 3D Gaussian Kernel of $8 \mathrm{~mm}$ at full-width-halfmaximum. 
Table 2. Exposure to recreational drugs (MATE2.1)

\begin{tabular}{|c|c|c|c|c|}
\hline & \multicolumn{4}{|c|}{ Exposure to recreational drugs } \\
\hline & $\begin{array}{l}\text { GHB-Coma }(n=24), \\
\text { mean } \pm \text { SD }\end{array}$ & $\begin{array}{l}\text { GHB-NoComa }(n=24) \\
\text { mean } \pm \text { SD }\end{array}$ & $\begin{array}{l}\text { No-GHB }(n=26) \\
\text { mean } \pm \text { SD }\end{array}$ & Difference, $p^{\mathrm{a}}$ value \\
\hline Alcohol & $11.44 \pm 31.87$ & $9.6 \pm 20.89$ & $12.45 \pm 34.84$ & 0.724 \\
\hline Nicotine & $105.81 \pm 141.14$ & $42.35 \pm 64.14$ & $41.13 \pm 84.89$ & 0.131 \\
\hline Cannabis & $3.70 \pm 6.61$ & $3.74 \pm 5.68$ & $4.15 \pm 6.48$ & 0.677 \\
\hline Cocaine & $1.90 \pm 5.34$ & $0.17 \pm 0.45$ & $0.03 \pm 0.12$ & $0.033^{*, 2}$ \\
\hline Stimulants & $3.76 \pm 7.98$ & $0.63 \pm 2.28$ & $0.16 \pm 0.38$ & $0.007^{*, 2,3}$ \\
\hline Ecstasy & $2.19 \pm 5.09$ & $0.10 \pm 0.34$ & $0.40 \pm 1.36$ & $0.016^{*, 1}$ \\
\hline Ketamine & $0.10 \pm 0.41$ & $0.22 \pm 0.92$ & $0.06 \pm 0.20$ & 0.987 \\
\hline Sedatives & $1.70 \pm 7.95$ & $0.18 \pm 0.82$ & $0.00 \pm 0.00$ & $0.003^{*, 1,2}$ \\
\hline \multicolumn{5}{|c|}{$\begin{array}{l}* p<0.05 . \\
{ }^{*} \text { Kruskal-Wallis. } \\
{ }^{1} \text { Post hoc analysis Mann-Whitney U: GHB-Coma }>\text { GHB-NoComa; ecstasy, } p=0.007 \text {; sedatives, } p=0.030 \text {. } \\
{ }^{2} \text { Post hoc analysis Mann-Whitney U: GHB-Coma }>\text { No-GHB; cocaine, } p=0.01 \text {; stimulants, } p=0.004 \text {; sedatives, } p=0.002 \text {. } \\
{ }^{3} \text { Post hoc analysis Mann-Whitney U: GHB-NoComa > No-GHB; stimulants, } p=0.015 \\
\text { GHB, gamma-hydroxybutyric acid. }\end{array}$} \\
\hline
\end{tabular}

The emotional and control conditions were modeled as box-car regressors and convolved with a canonical hemodynamic response function for each subject. To account for potential movement artefacts, realignment parameters were introduced as covariates of nointerest. Data were high-passed filtered $(1 / 128 \mathrm{~Hz})$, and temporal autocorrelation was modeled using an AR (1) process. Contrast images comparing emotional and control conditions were tested for group differences using analysis of covariance. As group differences were observed regarding IQ and exposure to cocaine, any other stimulants, ecstasy, and sedatives other than GHB (see results), these variables were introduced as covariates of no-interest throughout the analyses (IQ as a linear variable; exposure to the referenced drugs as 4 dummy variables). Two orthogonal planned comparisons were used to assess the effect of GHB-induced coma (contrast [a]; GHB-Coma > GHB-NoComa/No-GHB) and the effect of GHB-exposure (contrast [b]; GHB-NoComa > No-GHB). Voxel-wise statistical tests were family-wise-error (FWE) rate corrected $(p<0.05)$ for multiple comparisons at the cluster level (using a height threshold of $p<0.001$ ) across the whole brain or at peak level for regions of interest using a small volume correction (SVC). Four regions of interests for the left and right amygdala and the left and right hippocampus were anatomically defined using the Automated Anatomical Labeling atlas implemented within SPM12.

Since the main differences between groups were observed between the GHB-Coma group and the other 2 groups (see Results) and considering that the GHB-coma group consumed more GHB than the GHB-NoComa group, we also performed post hoc analyses to assess the effect of GHB-exposure and the effect of the number of GHB-induced comas on brain function within the GHBComa group. Since these clinical variables were positively skewed, we used a median-split approach and divided the GHB-Coma group in high and low GHB-exposure subgroups and in a high and low number of GHB-induced comas subgroups. Peak voxel parameter estimates of significant clusters were extracted from these subgroups and compared between them using 2-sample $t$ tests.

\section{Functional Connectivity}

To assess condition dependent functional connectivity, generalized psychophysiological interaction analyses were performed with the left hippocampus and the amygdala as seed regions [33]. The left hippocampus was selected as a seed region based on the results from the task-based group analysis and defined as a sphere of $5 \mathrm{~mm}$ radius around the peak activity of this analysis (coordinates: $-18,-38,4)$. The amygdala seed region was selected for its central role in affect processing and was defined as described above. Psychophysiological interaction images were obtained by multiplying the task regressors with the left hippocampus or the amygdala, providing differences in functional connectivity of the left hippocampus or the amygdala associated with the emotional and control conditions. Group differences were tested as described above.

\section{Results}

\section{Demographic and Clinical Characteristics}

Demographic and clinical characteristics of the overall sample are presented in Tables 1,2. No significant group differences were observed in age (mean $\pm \mathrm{SD}=26.49 \pm 6.9)$ and education level (mean $\pm \mathrm{SD}=5.14 \pm 1.5)$. Nevertheless, premorbid IQ was lower in the GHB-Coma group compared with the GHB-NoComa group $(p=0.004)$. As expected, the GHB-Coma group used more GHB than the GHB-NoComa group: higher daily doses of $\mathrm{GHB}(\mathrm{U}=111$, $p<0.001$ ), daily GHB-use during more months $(\mathrm{U}=194$, $p=0.013)$, and a tendency to use GHB more often during the last 30 days prior to the assessment $(U=219, p=0.044)$, though no group differences were observed in the duration 


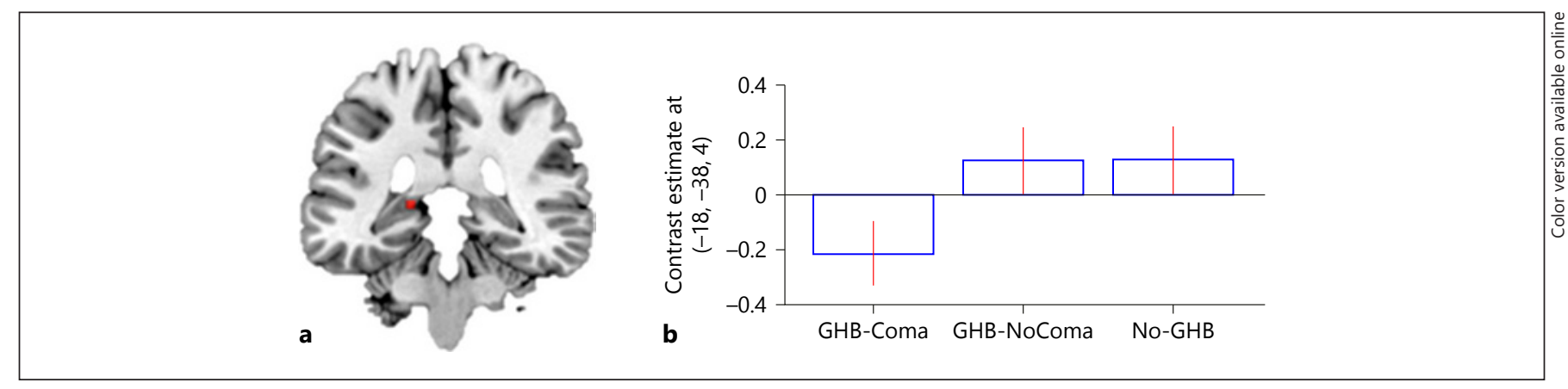

Fig. 1. Neural activity during matching of emotional faces (faces $>$ blurred ellipses) of the GHB-Coma group when compared with the GHB-NoComa and the No-GHB group (contrast a). Lower neural response of the hippocampus (a) in the GHB-Coma group when compared to the GHB-NoComa and the No-GHB groups, dis- played at $p<0.001$ uncorrected for visualization. The bar graph represents the contrast estimate with $90 \%$ confidence interval of the GHB-Coma, GHB-NoComa, and No-GHB groups on the hippocampus (b). GHB, gamma-hydroxybutyric acid.

the effect of multiple GHB-induced comas on the affective network (contrast a). The GHB-Coma group showed significantly lower recruitment of the left hippocampus when compared to the other 2 groups $\left(-18,-38,4 ; z=3.52 ; p_{\mathrm{svc}}=\right.$ 0.024; Fig. 1). No significant differences were observed when considering activity of the right hippocampus or the left and right amygdala. We then contrasted the GHB-NoComa group with the No-GHB group to assess the effect of GHB use per se (contrast b). This analysis showed no significant differences in activity between groups.

Next we assessed the effect of multiple GHB-induced comas on functional connectivity of the left hippocampus and the amygdala. This analysis showed higher functional connectivity of the left hippocampus with the left fusiform gyrus $\left(\mathrm{FG} ;-32,-46,-8 ; z=4.72 ; p_{\mathrm{FWE}}=0.005\right)$ and with a cluster in the right temporal-parietal-occipital junction (TPO-junction; $p_{\mathrm{FWE}}<0.001$ ) with 3 peak regions in the angular gyrus $(34,-62,44 ; z=4.34)$, the middle occipital gyrus $(40,-76,30 ; z=3.89)$, and the middle temporal gyrus $(48,-70,28 ; z=3.85)$ in the GHB-Coma group compared with the other 2 groups (Fig. 2). Considering the same contrast no significant group differences were observed for functional connectivity with the amygdala as seed region. When assessing the effect of GHB use per se, the results showed significantly lower hippocampal functional connectivity with the amygdala in the GHB-NoComa group compared with the No-GHB group $\left(30,4,-28 ; z=4.23 ; p_{\mathrm{SVC}}=0.001\right.$; Fig. 3$)$, but no significant differences were observed for this contrast when the amygdala was considered as the seed region.

Because most effects were observed for the difference between the GHB-Coma group compared with the other 2 groups, we performed a post hoc analysis to assess the 
Fig. 2. Left hippocampus functional connectivity of the GHB-Coma group when compared with the GHB-NoComa and the No-GHB groups (contrast a). Higher functional connectivity between the left hippocampus and (a) a region in the left FG and (c) a cluster in the right TPO-junction displayed at $p<0.001$ uncorrected for visualization purposes. The bar graphs represent the contrast estimate with $90 \%$ confidence interval for the GHB-Coma, the GHB-NoComa, and the No-GHB groups on the left FG (b) and the right TPO-junction (d). GHB, gamma-hydroxybutyric acid. TPOjunction, temporal-parietal-occipital junction; GHB, gamma-hydroxybutyric acid.

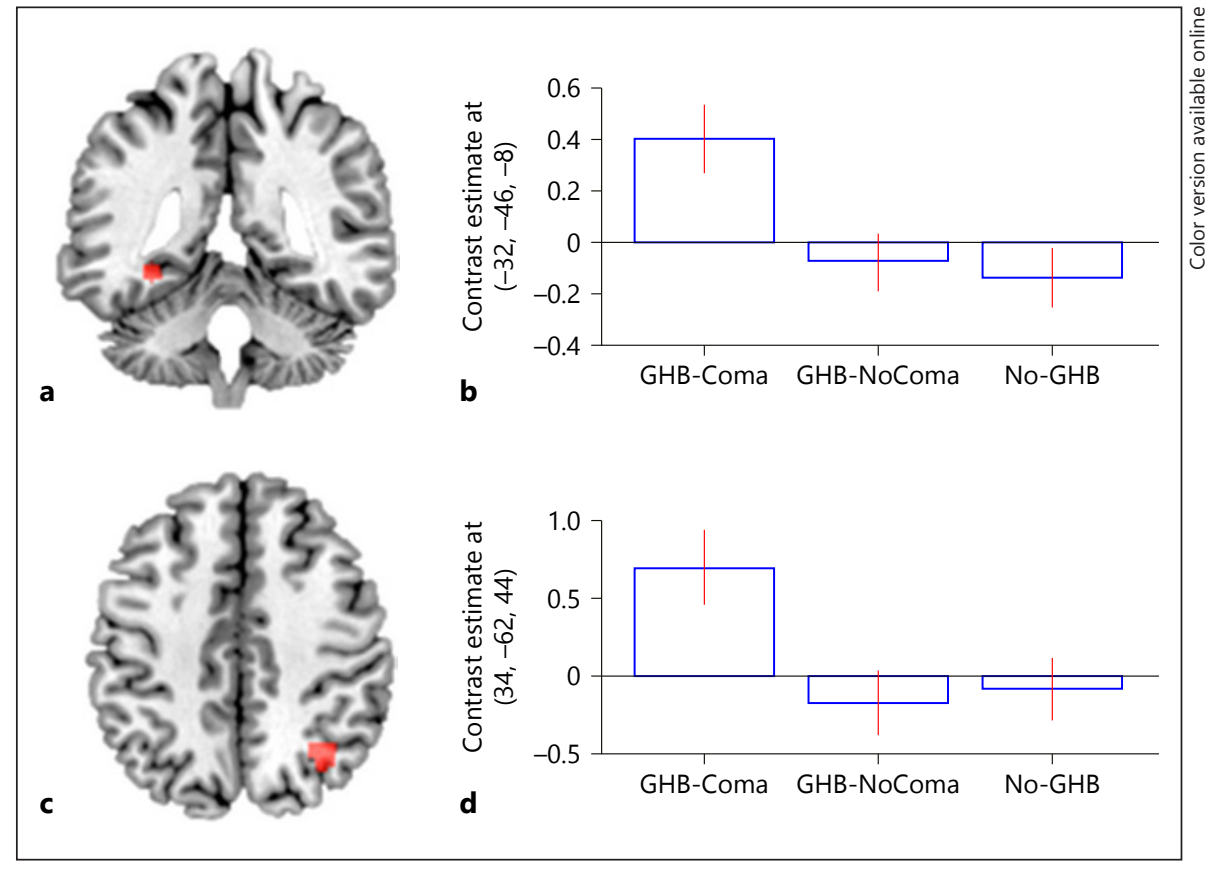

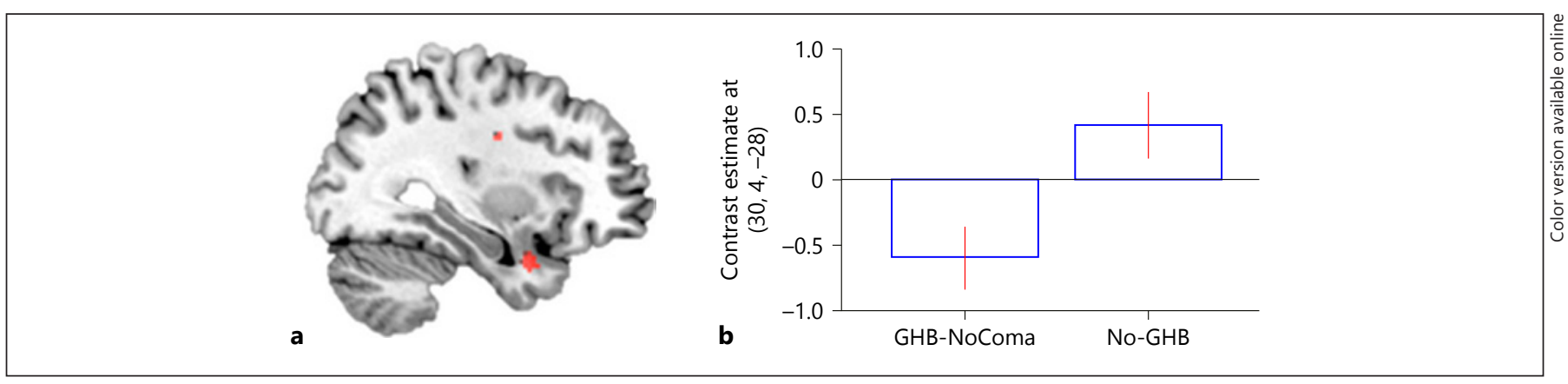

Fig. 3. Left hippocampus functional connectivity of the GHB-NoComa group when compared with the No-GHB group (contrast b). Lower functional connectivity between the left hippocampus and amygdala (a) displayed at $p<0.001$ uncorrected for visualiza- tion purposes. The bar graph represents the contrast estimate with $90 \%$ confidence interval for the GHB-NoComa and the No-GHB groups on the amygdala (b). GHB, gamma-hydroxybutyric acid. effect of GHB-exposure and the effect of the number of GHB-induced comas on brain function within the GHBComa group. Group comparisons between participants with high and low GHB-exposure or high and low number of GHB-induced comas showed no significant effects.

\section{Discussion}

In the current study, regular exposure to GHB was associated with increased negative affect and disruption of the affective network. This outcome appears to be largely driven by multiple GHB-induced comas, which were associated with increased depression, anxiety, and stress; lower hippocampal activity during matching of facial negative emotions; and higher left hippocampal functional connectivity with the left fusiform gyrus (FG) and a cluster on the right temporal-parietal-occipital junction (TPO-junction). The effect of GHB-use per se was associated with lower left hippocampal functional connectivity with the amygdala.

In controlled doses, GHB is known to decrease stress, social anxiety, and to induce a state of relaxation and sociability $[7,8,11]$. However, when taken regularly even at small controlled therapeutic doses, GHB might induce de- 
pression and increase anxiety in humans $[13,33]$. Furthermore self-reported levels of depression, anxiety, and stress are considerably higher in GHB-addicted patients who regularly take large concentrations of GHB $[11,12]$. Also, despite these levels being reduced after detoxification, they might persist for extended periods of time after cessation of the drug $[11,12]$. Here, the GHB-Coma group (regular users of higher concentrations of GHB when compared with the GHB-NoComa group) reported more negative feelings when compared with the other 2 groups. Regulation of negative feelings is associated with hippocampus and amygdala functioning [14]. Interestingly, the administration of GHB in animals leads to downregulation and altered function of the low-affinity GABA B receptor, highly presented in the hippocampus and in the amygdala [10,34]. Through these mechanisms, regular use of high-doses of GHB and multiple GHB-induced comas may lead to increased levels of depression, anxiety, and stress. However, no information on these levels before the occurrence of comas is available, and we cannot exclude the possibility that the reported differences in negative feelings were already present before the first coma had occurred. Conversely, these might have influenced the onset of recreational use of GHB, instead of resulting from its regular use.

While performing a matching task of negative emotions of fear and anger, the effect of multiple GHB-induced comas was further associated with decreased hippocampal activity, and increased left hippocampal functional connectivity with the left FG and a cluster in the right TPOjunction. During performance of this task, a matching process takes place between external stimuli and internal task goal representations stored in the hippocampus [35-37]. As a result, hippocampal activity tends to increase in order to activate task goal representations and allocate attention to high emotional salient stimuli that will enable an appropriate behavioral response $[37,38]$. However here, decreased hippocampal activity was instead observed in the GHB-Coma group. This might result from a disruption of hippocampal integrity, that may be associated with neurotoxicity linked to multiple GHB-induced comas $[14,15,37$, 39]. In line with this perspective, human studies investigating patients with hippocampal lesions have shown that disruption of its integrity results in the failure to produce appropriate emotion responses [17,39]. These effects are particularly pronounced when subjects are faced with stimuli of negative valence, which otherwise would induce reactivity of the hippocampus, or when demand upon cognitive function becomes too high $[16,17,40]$. Since our task required increased effort to discriminate between different negative emotional face stimuli, we hypothesize that the

Effect of Regular GHB Use on the

Affective Network lower activation of the left hippocampus observed in the GHB-Coma group represents blunted emotion identification of highly salient stimuli.

Moreover, when an emotion identification task requires emotion categorization, the activation of task goal representations stored in the hippocampus modulates visual interpretation regions such as the FG or the TPOjunction, in order to update and reorient attention toward the relevant salient emotional facial expression and ultimately provide the appropriate task response $[16,17$, $35,36]$. Though, if blunted responses to emotional stimuli are produced by the hippocampus, increased functional connectivity with visual interpretation areas likely operates as a compensatory mechanism to maintain adequate identification of such stimuli and determine their emotion valence $[17,35,39,40]$. In the GHB-Coma group, this potential compensatory mechanism likely allows the engagement of cognitive control systems required to achieve the same level of emotion identification performance as the other 2 groups.

Regarding our second hypothesis, GHB-use per se was associated with a decrease in functional connectivity between the left hippocampus and the amygdala. The hippocampus is responsible for storing emotion representations, which are used by the amygdala to prioritize emotional salient stimuli relevant for the current behavior $[17,38,40]$. Therefore, the GHB-related disruption of hippocampus-amygdala connectivity may further contribute to reduce allocation of attention toward relevant stimuli and thus attribute an inadequate emotion valence.

Furthermore, it should be noted that the majority of our results were observed with respect to multiple GHBinduced comas $[21,22]$. GHB-induced comas are often profound comas comparable to a state of general anesthesia. However, these comas are not accompanied by oxygen support and may lead to hypoxia and consequent oxidative stress [21]. In support of this assumption, neurotoxic effects have been associated with the effect of transient coma on the human brain [22-24]. Moreover, animal studies have shown a neurotoxic effect of GHB-induced sedation, particularly in the hippocampus, a region where disruptions in activity have also been associated with multiple GHB-induced comas in humans $[14,15,19]$. This cross-sectional study design does not allow us to make causal inferences regarding the link between GHB-use and the observed results. However, it is possible that GHB also has a neurotoxic effect in the human brain, which is likely exacerbated by multiple GHB-induced comas.

This study has both strengths and limitations. To the best of our knowledge, this is the first neuroimaging study as- 
sessing the effects of regular GHB use and multiple GHBinduced comas on the affect network. A particular strength of this study is the isolation of the effect of GHB use per se from the effect of multiple GHB-induced comas by including 2 groups matched for age and education level and controlling for IQ and exposure to recreational drugs other than GHB. Also, the inclusion of only male participants (although representative for the population of GHB users) limits the generalization of our findings [9]. Additionally, premorbid intellectual functioning was lower in the GHBComa group despite all groups being matched for education level. This may suggest a more generalized impairment of neurocognitive functioning. In order to minimize potential confounding by IQ, premorbid intellectual functioning was introduced as a covariate in all analyses. Also, no history of depression, anxiety, or stress was collected regarding early life and the period preceding the GHB-induced comas. This might be interesting to consider in future studies in order to understand if these levels reflect an underlying cause leading to regular use of higher recreational doses of GHB or are instead a result of the regular recreational use of this substance. We also did not include a healthy control group without a history of polydrug use, which did not allow us to investigate differences in brain function related to polydrug use per se. However, GHB users are often polydrug users, and we believe that the inclusion of a polydrug use control group in this preliminary study translates better to the reality of recreational GHB use. Nevertheless, all analyses were controlled for exposure to recreational use of drugs other than GHB, in order to minimize confounding. Future studies might benefit from the inclusion of a healthy drug-naïve control group to control for the possible influence of reward deficiency that is often observed in drug users and allow us to have a better estimate of the possible effects of GHB use per se. Prospective studies could further advance the existing knowledge about GHB-dependence and the causal link between GHB use and GHB-induced comas and the observed outcomes. Furthermore, it remains ambiguous what patterns of GHB use lead to GHB-induced comas, and would be beneficial to understand why some regular users using high doses of GHB never experience coma whereas others do. Therefore, in order to provide a more comprehensive view of the networks underlying GHB-induced coma, it would also be interesting to explore the influence of regular GHB-use and multiple GHB-induced comas in the amygdala and the hippocampus at a molecular level, by assessing gene expression and protein analysis in rat models. Finally, follow-up studies can potentially elucidate how reversible with time and abstinence are the brain alterations observed in this study.

\section{Conclusion}

Recreational use of GHB is associated with alterations in the affective network. The presence of multiple GHB-induced comas is related to increased levels of depression, anxiety, and stress, which are paralleled by decreased hippocampal activity and increased left hippocampal functional connectivity with visual interpretation areas. In addition, GHB use itself is related to decreased left hippocampal functional connectivity with the amygdala. These results suggest that GHB use and GHB-induced comas are not without risk, despite regular GHB-users despise the potential risk of GHB and multiple GHB-induced comas (often > 25 GHB-induced comas during prevalence). Awareness campaigns directed at recreational GHB users are warranted, to highlight the lasting adverse effects of GHB but especially the effect of GHB-induced comas, notwithstanding the absence of immediate apparent side effects.

\section{Acknowledgment}

The authors would like to thank Dr. Ruth van Holst for her help on the statistical analysis.

\section{Ethics Statement}

All participants have given their written informed consent to participate in this study.

The study protocol for this study has been approved by the Medical Ethics Review Committee of the Amsterdam Academic Medical Centre.

\section{Disclosure Statement}

The submitted manuscript has not been published previously, is not being considered for publication elsewhere, and has been approved by all authors. The authors have no conflicts of interest to declare.

\section{Funding Source}

This study was funded by the Dutch Ministry of Health, Welfare and Sport.

\section{Author Contributions}

M.T.B.M., and W.B.: designed the study. M.T.B.M., N.P., and Y.D.A.T.V.: collected the data. F.R.P., and G.A.W.: performed the statistical and neuroimaging analyses. F.R.P.: wrote the first draft of the manuscript. All authors revised and approved the final manuscript. 


\section{References}

1 Abanades S, Farré M, Segura M, Pichini S, Barral D, Pacifici R, et al. Gamma-hydroxybutyrate (GHB) in humans: pharmacodynamics and pharmacokinetics. Ann N Y Acad Sci. 2006 Aug;1074(1):559-76.

2 Korf DJ, Nabben T, Benschop A, Ribbink $\mathrm{K}$, van Amsterdam JG. Risk factors of $\gamma$-hydroxybutyrate overdosing. Eur Addict Res. 2014;20(2):66-74.

3 European Monitoring Centre for Drugs and Drug Addiction. European Drug Report Trends and Developments. Luxembourg: Publications Office of the European Union; 2018.

4 European Monitoring Centre for Drugs and Drug Addiction (EMCDDA). Hospital emergency presentations and acute drug toxicity in Europe: update from the Euro-DEN Plus research group and the EMCDDA. Luxembourg: Publications Office of the European Union; 2016.

5 Public Health England. Adult substance misuse statistics from the National Drug Treatment Monitoring System (NDTMS). 2016. Available from: www.gov.uk/phe.

6 United Nations Office on Drugs and Crime. World drug report - marketanalysis of synthetic drugs. United Nations publication; 2017.

7 Bosch OG, Esposito F, Havranek MM, Dornbierer D, von Rotz R, Staempfli P, et al. Gamma-Hydroxybutyrate Increases Resting-State Limbic Perfusion and Body and Emotion Awareness in Humans. Neuropsychopharmacology. 2017 Oct;42(11):2141-51.

8 Bosch OG, Havranek MM, Baumberger A, Preller KH, von Rotz R, Herdener M, et al. Neural underpinnings of prosexual effects induced by gamma-hydroxybutyrate in healthy male humans. Eur Neuropsychopharmacol. 2017 Apr;27(4):372-82.

9 Miró Ò, Galicia M, Dargan P, Dines AM, Giraudon $\mathrm{I}$, Heyerdahl $\mathrm{F}$, et al. Intoxication by gamma hydroxybutyrate and related analogues: clinical characteristics and comparison between pure intoxication and that combined with other substances of abuse. Toxicol Lett. 2017 Aug;277:84-91.

10 Johansson J. The Impact of Growth Hormone and Gamma-Hydroxybutyrate (GHB) on Systems Related to Cognition. Digit Compr Summ Uppsala Diss from Fac Pharm; 2012. p. 73.

11 Kamal RM, Dijkstra BA, de Weert-van Oene GH, van Duren JA, de Jong CA. Psychiatric comorbidity, psychological distress, and quality of life in gamma-hydroxybutyrate-dependent patients. J Addict Dis. 2017 Jan-Mar; 36(1):72-9.

12 Dijkstra BA, Kamal R, van Noorden MS, de Haan H, Loonen AJ, De Jong CA. Detoxification with titration and tapering in gammahydroxybutyrate $(\mathrm{GHB})$ dependent patients: the Dutch GHB monitor project. Drug Alcohol Depend. 2017 Jan;170:164-73.
13 xyrem group. A 12-month, open-label, multicenter extension trial of orally administered sodium oxybate for the treatment of narcolepsy. Sleep. 2003 Feb 1;26(1):31-5.

14 Pedraza C, García FB, Navarro JF. Neurotoxic effects induced by gammahydroxybutyric acid (GHB) in male rats. Int J Neuropsychopharmacol. 2009 Oct;12(9):1165-77.

15 van Nieuwenhuijzen PS, Long LE, Hunt GE, Arnold JC, McGregor IS. Residual social, memory and oxytocin-related changes in rats following repeated exposureto $\gamma$-hydroxybutyrate (GHB), 3,4-methylenedioxymethamphetamine (MDMA) or their combination. Psychopharmacology (Berl). 2010 Dec;212(4):663-74.

16 Adolphs R. Neural systems for recognizing emotion. Curr Opin Neurobiol. 2002 Apr; 12(2):169-77

17 Chudasama Y, Izquierdo A, Murray EA. Distinct contributions of the amygdala and hippocampus to fear expression. Eur J Neurosci. 2009 Dec;30(12):2327-37.

18 Raposo Pereira F, McMaster MT, Polderman $\mathrm{N}$, de Vries YD, van den Brink W, van Wingen GA. Effect of GHB-use and GHB-induced comas on dorsolateral prefrontal cortex functioning in humans. Neuroimage Clin. 2018; 20:923-30.

19 Raposo Pereira F, McMaster MT, Polderman $\mathrm{N}$, de Vries YD, van den Brink W, van Wingen GA. Adverse effects of GHB-induced coma on long-term memory and related brain function. Drug Alcohol Depend. 2018 Sep; 190:29-36.

20 Johansson J, Grönbladh A, Hallberg M. Gamma-hydroxybutyrate $(\mathrm{GHB})$ induces cognitive deficits and affects $G A B A B$ receptors and IGF- 1 receptors in male rats. Behav Brain Res. 2014 Aug;269:164-74.

21 van Amsterdam JG, Brunt TM, McMaster MT, Niesink RJ. Possible long-term effects of $\gamma$-hydroxybutyric acid (GHB) due to neurotoxicity and overdose. Neurosci Biobehav Rev. 2012 Apr;36(4):1217-27.

22 Perouansky M, Hemmings HC Jr. Neurotoxicity of general anesthetics: cause for concern? Anesthesiology. 2009 Dec;111(6):1365-71.

23 Nayak C, Nayak D, Raja A, Rao A. Time-level relationship between indicators of oxidative stress and Glasgow Coma Scale scores of severe head injury patients. Clin Chem Lab Med. 2006;44(4):460-3.

24 Snyder B, Shell B, Cunningham JT, Cunningham RL. Chronic intermittent hypoxia induces oxidative stress and inflammation in brain regions associated with early-stage neurodegeneration. Physiol Rep. 2017 May;5(9):e13258.

25 Carter LP, Pardi D, Gorsline J, Griffiths RR. Illicit gamma-hydroxybutyrate (GHB) and pharmaceutical sodium oxybate (Xyrem): differences in characteristics and misuse. Drug Alcohol Depend. 2009 Sep;104(1-2):1-10.

26 Büller HR, Fliers E, De Haes JCJM, Medema JP, Oude Elferink RPJ. Research code AMC independence in scientific research; 2010.
27 World Medical Association. World Medical Association Declaration of Helsinki: ethical principles for medical research involving human subjects. JAMA. 2013 Nov;310(20): 2191-4.

28 Schmand B, Bakker D, Saan R, Louman J. [The Dutch Reading Test for Adults: a measure of premorbid intelligence level]. Tijdschr Gerontol Geriatr. 1991 Feb;22(1):15-9.

29 Schippers GM, Broekman TG, Buchholz A. MATE-en 2.1 Manual and protocol-D. In: Cox WM, English edition. Nijmegen, Netherlands: Bur Bêta; 2011.

30 Lovibond SH, Lovibond PF. Manual for the Depression Anxiety Stress Scales. 2nd ed. Sydney: Psychology Foundation, 1995.

31 Hariri AR, Tessitore A, Mattay VS, Fera F, Weinberger DR. The amygdala response to emotional stimuli: a comparison of faces and scenes. Neuroimage. 2002 Sep;17(1): 317-23.

32 Crunelle CL, Kaag AM, van den Munkhof HE, Reneman L, Homberg JR, Sabbe B, et al. Dysfunctional amygdala activation and connectivity with the prefrontal cortex in current cocaine users. Hum Brain Mapp. 2015 Oct; 36(10):4222-30.

33 McLaren DG, Ries ML, Xu G, Johnson SC. A generalized form of context-dependent psychophysiological interactions (gPPI): a comparison to standard approaches. Neuroimage. 2012 Jul;61(4):1277-86.

34 Mamelak M, Swick T, Emsellem H, Montplaisir J, Lai C, Black J. A 12-week open-label, multicenter study evaluating the safety and patient-reported efficacy of sodium oxybate in patients with narcolepsy and cataplexy. Sleep Med. 2015 Jan;16(1):52-8.

35 Seghier ML. The angular gyrus: multiple functions and multiple subdivisions. Neuroscientist. 2013 Feb;19(1):43-61

36 Ciaramelli E, Grady CL, Moscovitch M. Topdown and bottom-up attention to memory: a hypothesis (AtoM) on the role of the posterior parietal cortex in memory retrieval. Neuropsychologia. 2008;46(7):1828-51.

37 Immordino-Yang MH, Singh V. Hippocampal contributions to the processing of social emotions. Hum Brain Mapp. 2013 Apr;34(4): 945-55.

38 Fastenrath M, Coynel D, Spalek K, Milnik A, Gschwind L, Roozendaal B, et al. Dynamic modulation of amygdala-hippocampal connectivity by emotional arousal. J Neurosci. 2014 Oct;34(42):13935-47.

39 Bisby JA, Horner AJ, Hørlyck LD, Burgess N Opposing effects of negative emotion on amygdalar and hippocampal memory for items and associations. Soc Cogn Affect Neurosci. 2016 Jun;11(6):981-90.

40 Lange K, Williams LM, Young AW, Bullmore ET, Brammer MJ, Williams SC, et al. Task instructions modulate neural responses to fearful facial expressions. Biol Psychiatry. 2003 Feb;53(3):226-32. 